\title{
Carence en vitamine B12 faisant évoquer des paresthésies buccales médicalement inexpliquées
}

\author{
Angelica Loup, Tommaso Lombardi, Jacky Samson * \\ Division de Stomatologie et Chirurgie orale, Rue Barthélémy-Menn 19, 1205 Genève, Suisse \\ *jacky.samson@unige.ch
}

Mots clés :

\section{carence en vitamine B12 / manifestations buccales / glossite de Hunter}

Key words:

vitamin B12 deficiency / oral manifestations / Hunter glossitis
Résumé - Dans les traités de Médecine, la glossite de Hunter constitue l'unique manifestation buccale de la carence en vitamine B12. En réalité, il existe bien d'autres manifestations mais, au stade initial, elles sont le plus souvent discrètes, polymorphes, intermittentes et d'installation insidieuse.

Abstract - Vitamin B12 deficiency mimiking oral paresthesias. In medical books, Hunter glossitis represents the only oral manifestation in vitamin B12 deficiency. In fact, there are some others manifestations but, at the inital stage, they are discret, polymorphic, intermittent, with insidious developement.

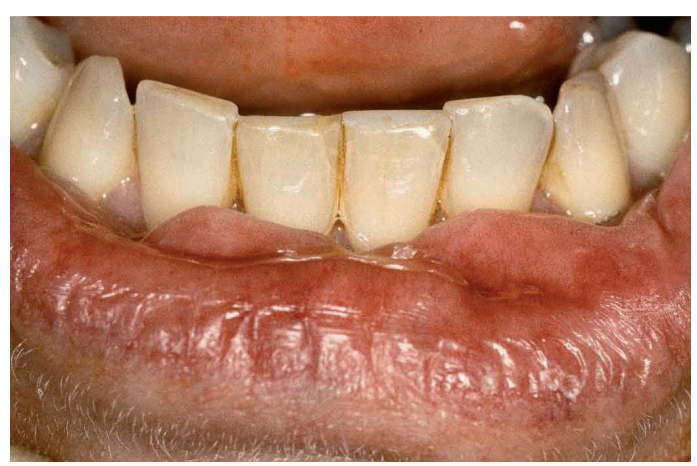

Fig. 1. Reliefs sur la lèvre inférieure secondaires à un tic de succion-aspiration nocturne.

Fig. 1. Lower double lip as side-effect of suckinginhaling tic at night.

Une patiente de 44 ans est venue consulter, sur les conseils de son médecin traitant, pour des brûlures de la muqueuse buccale évoluant depuis 1 mois. Elles sont discrètes mais présentes toute la journée, exacerbées par l'alimentation. Elle a reçu divers traitements (Mycostatine ${ }^{\circledR}$ et Valtrex ${ }^{\circledR}$ ) qui n'ont apporté aucune amélioration.

L'examen endo-buccal montrait deux lésions discrètes. Sur la face interne de la lèvre inférieure, près de la demimuqueuse, dans la région médiane, on observait deux reliefs, de section triangulaire, qui correspondaient à l'espace existant entre les incisives supérieures et les incisives inférieures lorsque la mandibule est en position de repos. Ces deux reliefs étaient secondaires à un tic de succion-aspiration nocturne (Fig. 1). La deuxième lésion était constituée par un érythème intéressant la moitié droite de la pointe de la langue; les papilles filiformes étaient conservées. La pauvreté du tableau clinique et le comportement de la patiente (patiente un peu agitée avec un tic de succion-aspiration nocturne) ont fait initialement suspecter des paresthésies buccales médicalement inexpliquées, jadis dites psychogènes. Toutefois, le caractère des douleurs ne correspondait pas à celles des paresthésies buccales médicalement inexpliquées : les brûlures étaient présentes toute la journée sans augmentation vespérale mais elles étaient exacerbées par l'alimentation. Les reliefs sur la muqueuse labiale inférieure existaient seulement depuis un mois et la patiente signalait que, depuis un mois aussi, elle ressentait une tension psychique inhabituelle, sans en trouver la raison. Ce tableau a fait évoquer une carence en vitamine B12. Un bilan biologique sommaire a été demandé. Il a révélé une anémie mégaloblastique (GR : 3,2 T/L ; VGM : $112 \mathrm{fL}$ ) avec un déficit en vitamine $B 12$ (84 pmol/L). La patiente a donc été ré-adressée à son médecin traitant pour des examens complémentaires et traitement. Ces investigations ont montré la présence d'anticorps anti-cellules pariétales augmentés $(320 ; n<10)$ et d'anticorps anti-facteur intrinsèque. La gastroscopie a révélé une modification de la muqueuse caractéristique d'une gastritechronique atrophique de type auto-immune, diagnostic qui a été confirmé par une biopsie. La patiente a été traitée par injection intramusculaire de vitamine B12 et dix jours après la 1ère infection l'érythème lingual, les brûlures et les reliefs labiaux avaient disparu. 


\section{Commentaires}

La vitamine B12 est fournie exclusivement par l'alimentation comportant des protéines animales (viande, abats, poissons...). Son absorption nécessite entre autres la présence de l'acide gastrique et du facteur intrinsèque (FI) sécrété par les cellules pariétales du fundus gastrique. L'apport quotidien varie entre 5 et $7 \mu \mathrm{g} / \mathrm{j}$ selon le régime alimentaire, sauf pour les végétaliens. Les besoins sont estimés entre 2 et $5 \mu \mathrm{g} / \mathrm{j}$ selon l'état physiologique (moins élevé chez l'enfant, plus élevés lors de la grossesse et l'allaitement). Environ $50 \%$ de la dose absorbée est stockée dans le foie principalement; la réserve hépatique varie entre 2 et $5 \mathrm{mg}$ ce qui représente environ $1000 \mathrm{j}$ d'apport. La vitamine B12 ingérée est complexée à des protéines alimentaires. Elle en est dissociée sous l'influence du suc gastrique (de l'acide chlorhydrique et de la pepsine surtout). Puis elle se lie aux haptocorrines, glycoprotéines porteuses présentes dans les sécrétions salivaires et gastriques. Dans le duodénum, ces protéines sont progressivement digérées et la vitamine B12 libérée se lie alors au FI, sous l'effet des sécrétions biliaires et pancréatiques. Le FI la protège du catabolisme bactérien iléal. Deux systèmes distincts contribuent à son absorption intestinale $[1,4,5]$. Le premier est spécifique et dépendant du FI, mais saturable : une succession de transports intraluminaux aboutit, dans l'iléon terminal, à la liaison du complexe vitamine B12 - FI à un récepteur cellulaire, la cubuline. Le second système d'absorption est indépendant du FI : il aboutit à l'absorption de $1 \%$ à $5 \%$ de la dose de vitamine B12 ingérée par simple diffusion. Il ne permet pas l'absorption d'une dose suffisante avec le régime alimentaire habituel mais, étant insaturable, on peut envisager un traitement substitutif per os. Après endocytose, la vitamine B12, dissociée du FI, se lie surtout à la transcobalamine II. Ce nouveau complexe, ou holotranscobalamine, passe dans le sang et transporte la vitamine B12 jusqu'aux cellules. Le cycle entéro-hépatique assure le stockage de la vitamine B12 dans le foie. Dans le rein, la mégaline, un récepteur tubulaire proximal rénal, permet la réabsorption de la vitamine B12 excrétée dans l'urine primitive. De ce fait, les réserves physiologiques en vitamine B12 sont abondantes : ceci explique l'installation tardive et insidieuse des signes cliniques (en moyenne 5-7 ans après l'arrêt de l'absorption).

On parle de carence en vitamine B12 lorsque le taux sérique est inférieur à $200 \mathrm{pg} / \mathrm{mL}$ (ou $150 \mathrm{pmol} / \mathrm{L}$ ) mais il existe une dissociation fréquente entre l'importance des anomalies biologiques (l'anémie en particulier) et la discrétion des manifestations cliniques. En conséquence, certains auteurs définissent la carence en vitamine B12 par la présence d'un taux sérique faible associé à des manifestations hématologiques et/ou cliniques. La vitamine B12 est une coenzyme ubiquitaire impliquée dans de nombreuses réactions enzymatiques intracellulaires. Elle intervient dans les réactions qui aboutissent à la synthèse de l'ADN et à celle de la méthionine à partir de l'homocystéine. L'altération de la synthèse de l'ADN entraîne un blocage de la maturation touchant d'abord les cellules à multiplication rapide d'où les manifestations hématologiques et muqueuses. L'altération de la synthèse de la méthionine à partir de l'homocystéine entraîne un défaut de synthèse de la protéine de base de la myéline, ce qui se traduit par des anomalies de la conduction nerveuse.

La maladie de Biermer a longtemps été considérée comme la principale cause de carence en vitamine B12. Le syndrome de non-dissociation de la vitamine B12 de ses protéines porteuses constitue maintenant l'étiologie la plus fréquente ; 10 à $30 \%$ des sujets âgés auraient une carence en vitamine B12 en raison de ce syndrome. La malabsorption, congénitale ou acquise, et la carence d'apport représentent des étiologies beaucoup plus rares. La carence en vitamine B12 comporte de nombreuses manifestations et chacune d'entre elles peut révéler l'affection [6]. Il s'agit de manifestations hématologiques (anémie mégaloblastique...), neuropsychiatriques (sclérose combinée de la moelle, troubles de l'humeur...), vasculaires (maladie thromboembolique...), digestives (flatulence, dyspepsie...) et muqueuses (glossite de Hunter...). En réalité, des manifestations stomatologiques polymorphes (des brûlures, un érythème, des érosions ou des lésions aphtoïdes pouvant intéresser la langue, les joues ou les lèvres; plus rarement une chéilite ou une perlèche), souvent discrètes, mais rebelles et récidivantes, peuvent précéder de plusieurs années les autres manifestations $[2,3]$. Les manifestations muqueuses sont secondaires à l'atrophie induite par la carence en vitamine B12 et régressent en 7 à 10 jours après le début du traitement.

Classiquement, pour le traitement substitutif, on prescrit des injections intra-musculaires; la posologie varie selon les pays (en France, $1000 \mu \mathrm{g}$ tous les 2-3 jours jusqu'à disparition des signes, puis $1000 \mu \mathrm{g}$ tous les mois); on a recours à la voie IV uniquement en cas d'urgence en raison du risque allergique. Plusieurs études récentes ont démontré que l'on peut également utiliser, avec la même efficacité, la voie per os (absorption par simple diffusion) sauf quand il existe des manifestations neuropsychiatriques; la posologie est de 1 à $2 \mathrm{mg} / \mathrm{j}$.

\section{Conflits d'intérêt : aucun}

\section{Références}

1. Andrès $E$, Mecili M, Ciobanu E. La vitamine B12 par voie orale à l'ère de l'évidence-based medecine. Med Therap 2010;16:5-11.

2. Campana F, Sibaud V, Taieb A, Fricain JC. Manifestations buccales révélatrices d'un déficit en vitamine $B 12$ : à propos d'un cas. Med Buccale Chir Buccale 2007;13:213-7.

3. Reygagne $P$, Kuffer $R$, Rybojad $M$, Dallot $A$, Vérola 0 , Brocheriou C. Maladie de Bierner révélée par des manifestations buccales et génitales. Ann Dermatol Venereol 1988;115:821-5.

4. Quadros EV. Advances in the understanding of cobalamin assimilation and metabolism. Br J Haematol 2009;148:195204.

5. Rufenacht $P$, Mach-Pascual S, Iten A. Hypovitaminose B12 : challenge diagnostique et thérapeutique. Rev Med Suisse 2008;4:2212-4, 16-7.

6. Serraj K, Mecili M, Andrès E. Signes et symptômes de la carence en vitamine B12 : revue critique de la littérature. Med Thérap 2010;16:13-20. 